\title{
Repetitive Learning Control of Nonlinear Continuous-Time Systems Using Quasi-Sliding Mode
}

\author{
Xiao-Dong Li, Tommy W. S. Chow, John K. L. Ho, and Hong-Zhou Tan
}

\begin{abstract}
In this brief, a quasi-sliding mode (QSM)-based repetitive learning control (RLC) method is proposed for tackling multi-input multi-output nonlinear continuous-time systems with matching perturbations. The proposed RLC method is able to perform rejection of periodic exogenous disturbances as well as tracking of periodic reference trajectories. It ensures a robust system stability when it is subject to nonperiodic uncertainties and disturbances. In this brief, an application to a robotic manipulator is used to illustrate the performance of the proposed QSM-based RLC method. A comparative study with the conventional variable structure control (VSC) technique is also included.
\end{abstract}

Index Terms-Nonlinear uncertain continuous-time systems, periodic exogenous disturbances, quasi-sliding mode (QSM), repetitive learning control (RLC).

\section{INTRODUCTION}

$\mathbf{P}$ ERIODIC signals are commonly experienced in industrial robotics, servo mechanisms, and other similar tracking scenarios, either in a form of reference inputs or disturbances. Such periodic signals include repetitive commands or operations for robotic control, and periodic disturbances of power supply [1]. This motivates a lot of work investigating different control methods for using the periodic nature of the mechanical dynamics. For repetitive operation of mechanical systems, an effective way to improve the performances is to "learn" the system behavior from its previous experience. Many types of repetitive learning control (RLC) methods have then been developed for compensating periodic exogenous disturbances and tracking periodic reference trajectories with a known period [1]-[13].

The RLC has been proven to be a useful method for asymptotic tracking or rejecting of periodic signals [6], [7], [11]. The basis of RLC relies on an internal model principle stating that a model of the external signal in the controller feedback loop provides asymptotic tracking and disturbance rejection [13]. Most RLC schemes are limited to linear systems [8], [12], [13], and

Manuscript received October 18, 2005. Manuscript received in final form September 19, 2006. Recommended by Associate Editor S. Saab. This work was supported in part by the National Natural Science Foundation of China under Grant 60575006

X.-D. Li was with the Department of Manufacturing Engineering and Engineering Management, City University of Hong Kong, Kowloon, Hong Kong. He is now with the Department of Electronics and Communication Engineering, Sun Yat-Sen University, 510275 Guangzhou, China (e-mail: xdlnx@263.net).

T. W. S. Chow is with the Department of Electronic Engineering, City University of Hong Kong, Kowloon, Hong Kong (e-mail: eetchow@ @ityu.edu.hk).

J. K. L. Ho is with the Department of Manufacturing Engineering and Engineering Management, City University of Hong Kong, Kowloon, Hong Kong (e-mail: mejohnho@ cityu.edu.hk).

H.-Z. Tan is with the Department of Electronics and Communication Engineering, Sun Yat-Sen University, 510275 Guangzhou, China (e-mail: issthz@mail.sysu.edu.cn).

Digital Object Identifier 10.1109/TCST.2006.886446 there has been very limited effort [3], [4] working on nonlinear RLC systems. It is also worth noting that virtually most major RLC results were obtained in single-input systems [2]-[4]. Interesting theoretical development of RLC can be found in [1] and [3]. Recently, various new techniques have been suggested for the RLC design. Among them, the variable structure control (VSC) technique with the prominent characteristics of invariance and robustness to parameter variations and exogenous disturbances has been successfully introduced to the design of RLC systems [2], [8]. In [8], the VSC and repetitive learning were used to improve tracking accuracy of a microactuator. The VSC part was used to stabilize the system, while the repetitive learning part was used to improve tracking accuracy. In [2], it designed an RLC controller using a discrete-time VSC methodology. It employed the notion of quasi-sliding mode (QSM) and the reaching law approach developed in [14] to design an RLC controller. But the control approach [2] was limited only to single-input single-output (SISO) linear discrete systems. There is, thus, a need to extend the QSM-based RLC approach to a broad level.

The concept of QSM in discrete systems was first proposed in [15]. It stated that the trajectory of the discrete VSC system in QSM would cross the switching plane upwards and forwards for a number of times. Gao et al. [14] further elaborated the QSM and proposed a reaching law approach for discrete system VSC design. In [16], a new QSM definition, which did not require the system state to cross the sliding hyperplane in each successive control step, and a novel QSM-based reaching law approach were proposed. It provided an improved performance in reducing both the chattering phenomenon and tracking errors.

In this brief, we focus on the design of RLC of nonlinear uncertain continuous-time systems because the investigation of RLC for continuous-time systems is essential in fundamental control theory. Our proposed RLC controller uses a modified discrete-time VSC methodology based on [16]. As there are two different dynamics in the RLC of nonlinear continuous-time systems, namely, the discrete iterative direction $k$, and the continuous time direction $t$, we derive the control rule from the convergence direction $k$ of the discrete QSM control method. As a result, periodic exogenous disturbances are eliminated or reduced. Tracking of periodic reference trajectories is also achieved. The proposed RLC approach ensures a robust stability of system dynamics to nonperiodic uncertainties and disturbances. This brief differs from the work of Sun et al. [2] and makes contributions mainly on these two aspects: 1) Our study extends the work described in [2] to multi-input multi-output (MIMO) nonlinear continuous-time systems with matching system uncertainties and exogenous disturbances and 2) corresponding to $m$ inputs of a control system, $m$ sub-QSMs are employed. 


\section{QSM AND REACHING CONDITION IN DISCRETE SYSTEMS}

Novel discrete QSM definition and a reaching condition have been derived for a special class of linear discrete systems with certain input matrix $(\Delta B=0)$ [16]. This section extends the QSM concept and the reaching condition to the following form of nonlinear single-input discrete system:

$$
x_{k+1}=F\left(x_{k}, u_{k}\right)
$$

where $F$ represents the nonlinear dynamics, and $x_{k} \in R^{n}$ and $u_{k} \in R$ are the state vector and input scalar, respectively. We denote the sliding function

$$
s_{k}=L\left(x_{k}\right)
$$

where $L$ represents a designed function; $s_{k} \in R$.

Definition 1: For nonlinear discrete system (1) and sliding function (2), we call the QSM in the $\varepsilon$ vicinity of the sliding hyperplane $s_{k}=0$ such a motion of the system in direction $k$ that

$$
\left|s_{k}\right| \leq \varepsilon
$$

where the positive constant $\varepsilon$ is called the QSM bandwidth.

Definition 2: Nonlinear discrete system (1) satisfies the reaching condition of the QSM (3) if and only if for any $k \geq 0$, there are

$$
\begin{gathered}
s_{k}>\varepsilon \Rightarrow-\varepsilon \leq s_{k+1}<s_{k} \\
s_{k}<-\varepsilon \Rightarrow s_{k}<s_{k+1} \leq \varepsilon \\
\left|s_{k}\right| \leq \varepsilon \Rightarrow\left|s_{k+1}\right| \leq \varepsilon .
\end{gathered}
$$

In [16], a reaching law approach was proposed for linear discrete SISO systems using the previous QSM definition and reaching conditions. As a result, the maximum distance of the system state from the sliding plane $s_{k}=0$ after convergence is less than one-half of the same distance for the system controlled according to the algorithm introduced in [14].

\section{QSM-BASED RLC FOR NONLINEAR CONTINUOUS-TIME SYSTEMS}

\section{A. Problem Formulation}

Consider the following MIMO nonlinear uncertain continuous-time system performing repetitive tasks over a fixed-time interval $t \in[0, T]$

$$
\dot{x}_{k}(t)=A\left(x_{k}(t), t\right)+B\left(x_{k}(t), t\right) \cdot\left[u_{k}(t)+w_{k}\left(x_{k}(t), t\right)\right]
$$

where $k$ denotes the $k$ th repetitive operation of the system. For all $t \in[0, T], x_{k}(t) \in R^{n}$, and $u_{k}(t) \in R^{m}(m<n)$ are the state (output) vector and the input vector, respectively; $A\left(x_{k}(t), t\right) \in R^{n}$ and $B\left(x_{k}(t), t\right) \in R^{n \times m}$ are the nominal variable vector/matrix; $w_{k}\left(x_{k}(t), t\right) \in R^{m}$ represents the lumped matching uncertainties and exogenous disturbances. It is assumed that $B\left(x_{k}(t), t\right)$ is a full-column-rank matrix for all $t$ and $k$. Nonlinear uncertain continuous-time system (4) achieves to track a differentiable periodic reference trajectory $x_{d}(t) \in$ $R^{n}$ over $[0, T]$, where $x_{d}(t)$ is closed, i.e., $x_{d}(0)=x_{d}(T)$. The initial condition of (4) at the beginning of each cycle is assumed to be coherent with the terminal state of the preceding cycle such that $x_{k+1}(0)=x_{k}(T)$ for $k=0,1,2, \ldots$. For some control applications with a repetitive task, there may be a mismatch in initial condition $x_{k+1}(0)=x_{k}(T)$ [17]. The mismatch is not considered in our RLC design. To design an QSM-based RLC to track the periodic reference trajectory $x_{d}(t)$, a filtered tracking error at the $k$ th cycle

$$
s_{k}(t)=C\left(x_{k}(t)-x_{d}(t)\right)
$$

is assigned for each $t \in[0, T]$, where $C \in R^{m \times n}$ is a specified full-row-rank matrix. On the selection of $C$, different techniques can be used to ensure the required properties [19]. For example, we can transform systems (4) into a reduced form, a controllability form, or a sliding mode equivalent system for determining the matrix $C$ based on the transformed form. From (5) and the initial condition of system (4), we have $s_{k}(T)=s_{k+1}(0)$ for all $k=0,1,2, \ldots$ Thus, the objective of the RLC is to iteratively determine the control input $u_{k}(t)$, i.e., $u_{k+1}(t)=G\left(u_{k}(t)\right)$, such that as $k \rightarrow \infty$, the filtered tracking error $s_{k}(t)$ can be driven into a small bounded region (as small as possible) by rejecting the periodic component of exogenous disturbance.

For convenience of mathematical expression in this brief, for any $m$ dimensional vectors $\alpha=\left[\alpha^{(1)} \alpha^{(2)}, \ldots, \alpha^{(m)}\right]^{T}$ and $\beta=\left[\beta^{(1)} \beta^{(2)}, \ldots, \beta^{(m)}\right]^{T}, \beta^{(1)}, \beta^{(2)}, \ldots, \beta^{(m)}>0$, we denote $|\alpha| \leq \beta$ if and only if $\left|\alpha^{(i)}\right| \leq \beta^{(i)}$ for all $i=1,2, \ldots, m$. We let

$$
\begin{aligned}
& d_{k}(t)=C\left[B\left(x_{k+1}(t), t\right) w_{k+1}\left(x_{k+1}(t), t\right)\right. \\
&\left.-B\left(x_{k}(t), t\right) w_{k}\left(x_{k}(t), t\right)\right]
\end{aligned}
$$

where $d_{k}(t)=\left[d_{k}^{(1)}(t) d_{k}^{(2)}(t), \ldots, d_{k}^{(m)}(t)\right]^{T}$. In the RLC process of (5), it is assumed that the uncertainty and exogenous disturbance are bounded so that the following relation holds for $k \geq k^{*}$ :

$$
\left|\int_{0}^{t} d_{k}(\tau) d \tau\right| \leq \delta \quad t \in[0, T]
$$

where $\delta=\left[\delta^{(1)} \delta^{(2)}, \ldots, \delta^{(m)}\right]^{T}$, and $k^{*}$ is a sufficient large integer.

\section{B. QSM-Based RLC Rule}

In order to derive the required QSM-based RLC rule, let us modify the filtered tracking error $s_{k}(t)$ and construct a sliding function on a discrete index $k$

$$
\bar{s}_{k}(t)=s_{k}(t)-\theta_{h}(t)\left(\frac{t}{h}-1\right)^{2} s_{k}(0)
$$

where $\theta_{h}(t)=\left\{\begin{array}{ll}1, & t \in[0, h] \\ 0, & t \in(h, T]\end{array}\right.$, and $h$ is a selected value between 0 and $T$. Clearly, we have $\bar{s}_{k}(0)=0$. For each $t \in[0, T]$, a sliding hyperplane in iterative direction $k$ is, thus, defined as

$$
\bar{s}_{k}(t)=0 \text {. }
$$

Let $\bar{s}_{k}(t)=\left[\bar{s}_{k}^{(1)}(t) \bar{s}_{k}^{(2)}(t), \ldots, \bar{s}_{k}^{(m)}(t)\right]^{T}$. Equation (9) includes $m$ sliding sub-hyperplanes $\bar{s}_{k}^{(i)}(t)=0, i=1,2, \ldots, m$.

In this section, in order to match the MIMO nonlinear uncertain continuous-time system (4), for each $t \in[0, T]$, we employ 
the QSM results of the last section to MIMO systems, thereby $m$ sub-QSMs in direction $k$ corresponding to $m$ sub-hyperplanes $\bar{s}_{k}^{(i)}(t)=0, i=1,2, \ldots, m$, are defined as follows:

$$
\left|\bar{s}_{k}^{(i)}(t)\right| \leq \varepsilon_{i} \quad i=1,2, \ldots, m
$$

where the positive constant $\varepsilon_{i}$ is the $i$ th QSM bandwidth. Under the previous sub-QSM definition (10), we propose the following reaching law in discrete direction $k$ for the QSM control:

$$
\bar{s}_{k+1}^{(i)}(t)=r_{k+1}^{(i)}(t)+\int_{0}^{t} d_{k}^{(i)}(\tau) d \tau \quad t \in[0, T], i=1,2, \ldots, m
$$

where $r_{k}^{(i)}(t)$ is defined as follows.

If $\left|\bar{s}_{0}^{(i)}(t)\right|>2 \delta^{(i)}$, then, let $0<\bar{k}^{(i)}(t)<\left(\left|\bar{s}_{0}^{(i)}(t)\right| / 2 \delta^{(i)}\right)$, and $r_{k}^{(i)}(t)=\left(\left(\bar{k}^{(i)}(t)-k\right) / \bar{k}^{(i)}(t)\right) \bar{s}_{0}^{(i)}(t)$ for $k<\bar{k}^{(i)}(t)$, $r_{k}^{(i)}(t)=0$ for $k \geq \bar{k}^{(i)}(t)$. (In our simulation studies, we uniformly set $\bar{k}^{(i)}(t)=\operatorname{int}\left(\left(\left|\bar{s}_{0}^{(i)}(t)\right| / 4 \delta^{(i)}\right)+1\right)$, where $\operatorname{int}($.$) rep-$ resents the integer part of value.) Otherwise, i.e., if $\left|\bar{s}_{0}^{(i)}(t)\right| \leq$ $2 \delta^{(i)}$, then $r_{k}^{(i)}(t)=0$ for $k \geq 0$.

The reaching law (11) together with the definition of $r_{k}^{(i)}(t)$ specifies the dynamics of a sliding function $\bar{s}_{k}^{(i)}(t)$, which implies that the reaching condition of sub-QSM with a bandwidth $\delta^{(i)}$ in Section II can be satisfied. Also, the dynamics of $\bar{s}_{k}^{(i)}(t)$ can be controlled by choosing the parameter $\bar{k}^{(i)}(t)$. Let $r_{k}(t)=\left[r_{k}^{(1)}(t) r_{k}^{(2)}(t), \ldots, r_{k}^{(m)}(t)\right]^{T}$. From the initial condition of sliding function $\bar{s}_{k}(0)=0$ and the definition of $r_{k}^{(i)}(t)$, we get $r_{k}(0)=0$ for all $k$.

From the reaching law (11), we have

$$
\dot{\bar{s}}_{k+1}(t)=\dot{r}_{k+1}(t)+d_{k}(t) \quad t \in[0, T] .
$$

On the other hand, consider the difference between $\dot{\bar{s}}_{k+1}(t)$ and $\dot{\bar{s}}_{k}(t)$ expressed as

$$
\begin{aligned}
\dot{\bar{s}}_{k+1}(t)-\dot{\bar{s}}_{k}(t)= & C\left[A\left(x_{k+1}(t), t\right)-A\left(x_{k}(t), t\right)\right] \\
& +C B\left(x_{k+1}(t), t\right) u_{k+1}(t) \\
& -C B\left(x_{k}(t), t\right) u_{k}(t)+d_{k}(t) \\
& -\theta_{h}(t) \frac{2}{h}\left(\frac{t}{h}-1\right) \times\left[s_{k+1}(0)-s_{k}(0)\right] .
\end{aligned}
$$

Then, combining (12) and (13), an RLC rule is obtained as follows:

$$
\begin{aligned}
u_{k+1}(t)=( & \left.C B\left(x_{k+1}(t), t\right)\right)^{-1} \\
\times & \left\{C B\left(x_{k}(t), t\right) u_{k}(t)\right. \\
& \quad-C\left[A\left(x_{k+1}(t), t\right)-A\left(x_{k}(t), t\right)\right]-\dot{s}_{k}(t) \\
& \left.+\dot{r}_{k+1}(t)+\theta_{h}(t) \frac{2}{h}\left(\frac{t}{h}-1\right) \cdot s_{k+1}(0)\right\} .
\end{aligned}
$$

Theorem 1: Given the nonlinear uncertain continuous-time system (4) performing a periodic reference trajectory tracking at a finite time interval $T$, the uncertainty and exogenous disturbance satisfy the condition (7), and the periodic reference trajectory $x_{d}(t)$ is differentiable. Then, using the RLC rule (14), we have $\lim _{k \rightarrow \infty}\left|s_{k}(t)\right| \leq \delta$ for $t \in[h, T]$, where the bound $\delta$ is defined in (7).

Proof: When the QSM-based RLC rule (14) is applied to the nonlinear uncertain continuous-time system (4), we have (12). Integrating the two sides of (12), and considering that $\bar{s}_{k}(0)=0$ and $r_{k}(0)=0$ for all $k$, we obtain the same QSM dynamics as (11). Let $k^{* *}=\max _{t, i} \bar{k}^{(i)}(t)$. From the definition of $r_{k}^{(i)}(t)$ and (11), it can be derived that as $k \geq k^{* *}$, we have

$$
\bar{s}_{k+1}(t)=\int_{0}^{t} d_{k}(\tau) d \tau \text {. }
$$

Therefore, from the condition (7), $\left|\bar{s}_{k}(t)\right| \leq \delta$ for $t \in[0, T]$ and $k>\max \left\{k^{*}, k^{* *}\right\}$. On the other hand, from (8) and the definition of $\theta_{h}(t)$, it is noticed that $\bar{s}_{k}(t)=s_{k}(t)$ at $t \in[h, T]$. Therefore, we can conclude that $\left|s_{k}(t)\right| \leq \delta$ for $t \in[h, T]$ and $k>\max \left\{k^{*}, k^{* *}\right\}$. End of proof.

Remark 1: From the previous proof process, we have $\left|s_{k}(t)\right| \leq \delta$ for $t \in[h, T]$ and $\left|s_{k+1}(0)\right|=\left|s_{k}(T)\right| \leq \delta$ as $k>\max \left\{k^{*}, k^{* *}\right\}$. If $h$ is sufficiently small, it can be further concluded that $\left|s_{k}(t)\right| \leq \delta$ for all $t \in[0, T]$ and $k>\max \left\{k^{*}, k^{* *}\right\}+1$ because of the continuity of $s_{k}(t)$ on $t$.

Remark 2: The RLC rule (14) can be expressed as the following form:

$$
\begin{aligned}
& u_{k+1}(t)=-\hat{w}_{k+1}(t)+\left(C B\left(x_{k+1}(t), t\right)\right)^{-1} \\
& \times\left[-C A\left(x_{k+1}(t), t\right)+C \dot{x}_{d}(t)+\dot{r}_{k+1}(t)\right. \\
& \left.+\theta_{h}(t) \frac{2}{h}\left(\frac{t}{h}-1\right) s_{k+1}(0)\right]
\end{aligned}
$$

where $\hat{w}_{k+1}(t)=\left(C B\left(x_{k+1}(t), t\right)\right)^{-1}\left[C \dot{x}_{k}(t)-C A\left(x_{k}(t), t\right)\right.$ - $\left.C B\left(x_{k}(t), t\right) u_{k}(t)\right]$ is the estimation of $w_{k+1}\left(x_{k+1}(t), t\right)$. This indicates that the RLC rule (14) provides disturbance compensation even though no disturbance estimate is explicitly used.

Remark 3: It is worth noting that the computation of the RLC rule (14) requires knowledge on the state vector $x_{k}(t)$ of the RLC system. We need an additional observer if it cannot be obtained.

Remark 4: Furthermore, if there is no uncertainty to the system (4), and the exogenous disturbance is periodic with time interval $T$, i.e., $w_{k+1}\left(x_{k+1}(t), t\right)=w_{k}\left(x_{k}(t), t\right)=w(t)$, then, the following can be derived from (15):

$$
\begin{array}{r}
s_{k+1}(t)=\int_{0}^{t} C\left[B\left(x_{k+1}(\tau), \tau\right)-B\left(x_{k}(\tau), \tau\right)\right] w(\tau) d \tau, \\
t \in[h, T] .
\end{array}
$$

In many periodic reference trajectory tracking servo mechanism and robotic systems, $s_{k}(t)$ enters and remains within the 
bound $\delta . B\left(x_{k+1}(t), t\right)$ and $B\left(x_{k}(t), t\right)$ are, thus, very close that $s_{k+1}(t)$ approaches to zero from (17). Therefore, the RLC rule (14) in these mechanistic systems can reject periodic exogenous disturbance. This effect will be illustrated by the later simulations presented in this brief. Especially, if $B\left(x_{k}(t), t\right)=B(t)$ in the nonlinear uncertain continuous-time system (4), a complete rejection to periodic exogenous disturbance is achievable. This is demonstrated by the following Corollary 1.

Corollary 1: Given the MIMO nonlinear uncertain continuous-time system

$$
\dot{x}_{k}(t)=A\left(x_{k}(t), t\right)+B(t)\left(u_{k}(t)+w_{k}(t)\right)
$$

in order to perform a periodic reference trajectory tracking at a finite time interval $T$, the exogenous disturbance satisfies the periodic condition $w_{k+1}(t)=w_{k}(t)=w(t)$ for $t \in[0, T]$, and the periodic reference trajectory $x_{d}(t)$ is differentiable. Then, using the following RLC rule:

$$
\begin{aligned}
& u_{k+1}(t) \\
& =u_{k}(t)+(C B(t))^{-1} \\
& \quad \times\left\{C\left[A\left(x_{k}(t), t\right)-A\left(x_{k+1}(t), t\right)\right]\right. \\
& \left.\quad-\dot{s}_{k}(t)+\dot{r}_{k+1}(t)+\theta_{h}(t) \frac{2}{h}\left(\frac{t}{h}-1\right) \cdot s_{k+1}(0)\right\}
\end{aligned}
$$

we have $\lim _{k \rightarrow \infty} s_{k}(t)=0$ for $t \in[0, T]$.

Proof: Based on the proof of Theorem 1 and the periodic exogenous disturbance $w_{k+1}(t)=w_{k}(t)=w(t)$ for $t \in[0, T]$, when the QSM-based RLC rule (19) is applied to the nonlinear uncertain continuous-time system (18), we have $\bar{s}_{k+1}(t)=\int_{0}^{t} d_{k}(\tau) d \tau=0$ for $t \in[0, T]$ and $k \geq k^{* *}$. Therefore, we obtain

$$
s_{k+1}(t)=\theta_{h}(t)\left(\frac{t}{h}-1\right)^{2} s_{k+1}(0) \quad t \in[0, T], k \geq k^{* *} .
$$

We have $s_{k}(t)=0$ for $t \in[h, T]$ and $k>k^{* *}$ from (20) and the definition of $\theta_{h}(t)$. Moreover, from (20) and the initial repetitive condition $s_{k+1}(0)=s_{k}(T)=0$, we have $s_{k+1}(t)=0$ for all $t \in[0, T]$ and $k>k^{* *}+1$. That is, $\lim _{k \rightarrow \infty} s_{k}(t)=0$ for $t \in[0, T]$. End of proof.

\section{Simulations}

We use the arm dynamics of a two-link robotic shown in Fig. 1 as an example to demonstrate the performance of the proposed RLC method. Comparative study with the VSC technique is also included. In practice, a robotic manipulator often operates at situations that reference commands to be tracked and/or disturbance inputs to be rejected are both in a form of periodic signal. Its dynamics are described by

$$
M(\theta) \ddot{\theta}+n(\theta, \dot{\theta})=\tau+w\left(\theta, p, t^{\prime}\right)
$$

where $\theta=\left[\theta^{(1)} \theta^{(2)}\right]^{T}$ and $\theta^{(1)}$ and $\theta^{(2)}$ are absolute joint angles; $\tau=\left[\tau^{(1)} \tau^{(2)}\right]^{T}$ and $\tau^{(1)}$ and $\tau^{(2)}$ are control torques; $t^{\prime} \in[0, \infty)$ is the time variable; $w\left(\theta, p, t^{\prime}\right)$ is the collection of

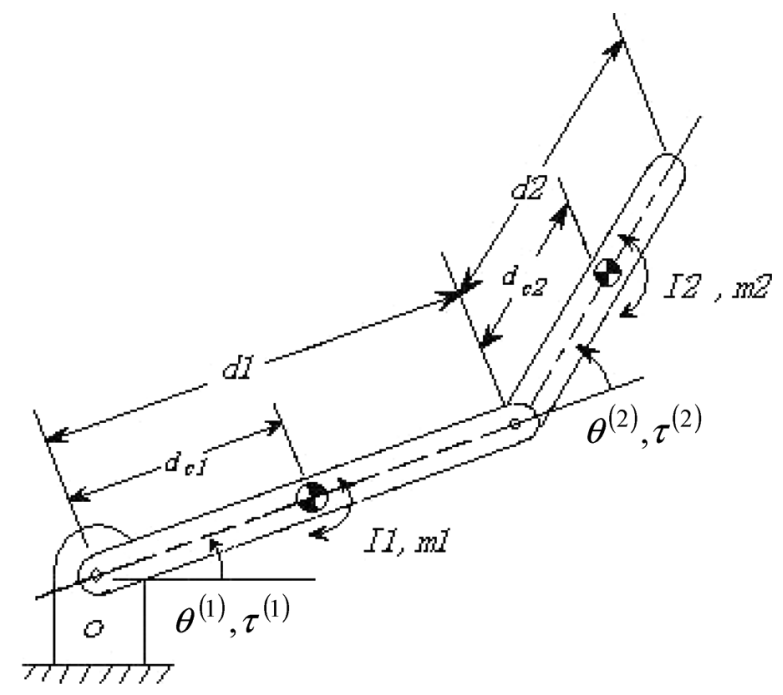

Fig. 1. Geometry of a two-link robotic arm.

all uncertainties and disturbances. Further details of (21) of the two-link robotic can be found in [18]. In the robotic arm dynamics (21), the gravitational effect is neglected, and the perturbation $w\left(\theta, p, t^{\prime}\right)$ is bounded. In our simulation, the arm masses $m 1$ and $m 2$ are set as 20 and $10 \mathrm{~kg}$, respectively; the arm lengths $d 1$ and $d 2$ are set as $1 \mathrm{~m}$; the distances of mass center $d_{c 1}$ and $d_{c 2}$ are set as $0.5 \mathrm{~m}$; the moments of inertia $I 1$ and $I 2$ are set as 0.8 and $0.2 \mathrm{~kg} \cdot \mathrm{m}^{2}$, respectively. We define a 4-D state vector $x$ as $x=[\theta \dot{\theta}]^{T}$, then (21) is in the form

$$
\dot{x}=A(x)+B(x) \cdot(u+w)
$$

where $u=\tau, A(x)=\left[\begin{array}{c}\dot{\theta} \\ -M^{-1}(x) n(x)\end{array}\right], B(x)=\left[\begin{array}{c}0 \\ M^{-1}(x)\end{array}\right]$. The simulation was conducted to track the periodic reference trajectories of the robotic joint angles expressed as

$$
\begin{aligned}
& \theta_{d}^{(1)}=\left(0.8+0.2 \sin \left(0.25 \pi t^{\prime}\right)\right) \sin \left(0.5 \sin \left(0.25 \pi t^{\prime}\right)\right) \\
& \theta_{d}^{(2)}=\left(0.6+0.2 \sin \left(0.25 \pi t^{\prime}\right)\right) \sin \left(0.5 \sin \left(0.25 \pi t^{\prime}\right)\right)
\end{aligned}
$$

with period $T=8$. We define the joint angle errors $e^{(i)}$ and the filtered tracking errors $s^{(i)}$ as

$$
e^{(i)}=\theta^{(i)}-\theta_{d}^{(i)} \quad s^{(i)}=3 e^{(i)}+\dot{e}^{(i)} \quad(i=1,2) .
$$

The filtered matrix $C=\left(\begin{array}{cccc}3 & 0 & 1 & 0 \\ 0 & 3 & 0 & 1\end{array}\right)$. Let $t^{\prime}=k T+t$, and $t \in[0, T]$, (22) can be expressed as the form of (4). In the RLC process of nonlinear uncertain continuous-time system (22), the initial control conditions $x(0)=\left[\begin{array}{lllll}0 & 0 & 0.3 & 0.3\end{array}\right]^{T}$, and $u(t)=\left[\begin{array}{ll}0 & 0\end{array}\right]^{T}$ for $t \in[0,8)$ are assumed. A digital controller of RLC rule (14) with $\delta=[0.20 .4]^{T}$ and $h=1$ is used with the sampling time set as $10^{-2} \mathrm{~s}$. We need to discuss the following two cases.

1) The perturbation $w$ is assumed to be periodic given as $\omega=\left[2 \sin \left(\pi t^{\prime}\right) 2 \sin \left(\pi t^{\prime}\right)\right]^{T}$. It has the same period as the desired trajectory $\theta_{d}^{(i)}$. For $i=1,2$, the tracking errors $e^{(i)}$ (in radians) of the joint angles $\theta^{(i)}$ are shown in Fig. 2, and the relevant control torques $\tau^{(i)}$ (in Newton-meters) at time interval $[60,120]$ is shown in Fig. 3. In Fig. 2, the absolute value of the tracking errors $e^{(i)}$ are driven to less 


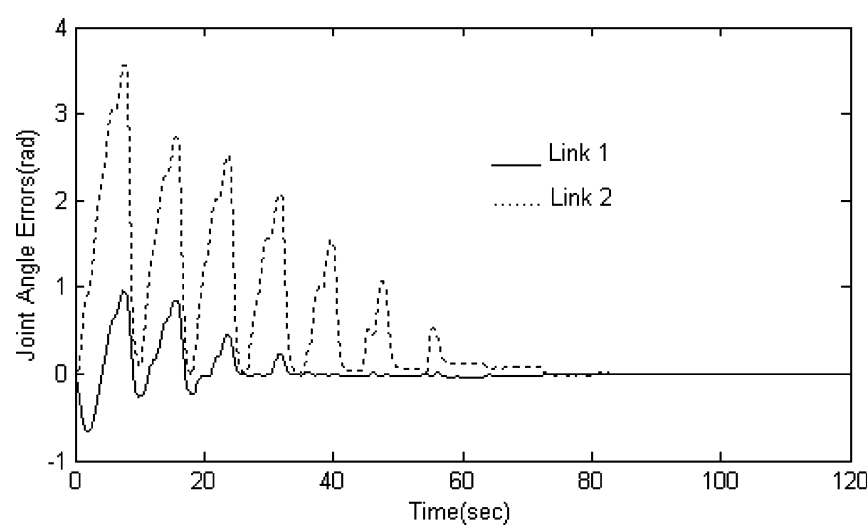

Fig. 2. Joint angle errors obtained by the proposed RLC method under periodic perturbation.

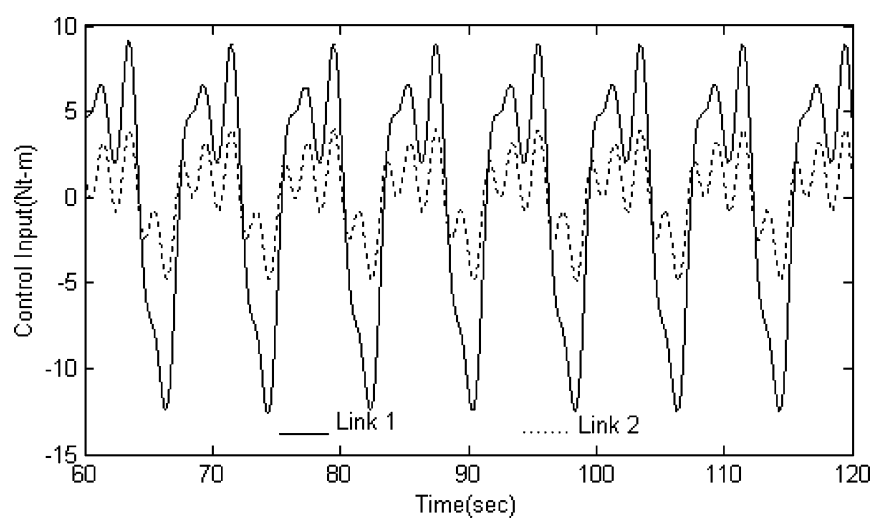

Fig. 3. Control torques at the time interval $[60,120]$ obtained by the proposed RLC method under periodic perturbation.

than $6 \times 10^{-4}$ as $k>14$. In order to demonstrate the effect of rejecting periodic exogenous disturbance when tracking periodic reference trajectories, we use an index $E_{k}=\max _{t \in[0, T]}\left\|B\left(x_{k+1}(t)\right)-B\left(x_{k}(t)\right)\right\|_{F}$ on repetitive cycle $k$, where $\|\cdot\|_{F}$ denotes the Frobenius norm of the matrix. Fig. 4 shows how $E_{k}$ varies with repetitive cycle $k$. In this simulation, $E_{k}$ is driven to less than $4 \times 10^{-4}$ as $k>14$. Remark 4 on Theorem 1 is then verified. On the other hand, in order to further demonstrate the advantages of the proposed RLC method against periodic perturbation, the conventional VSC technique (49) [19] for MIMO nonlinear continuous-time systems is employed for comparative study. The parameters are set as $q_{1}=q_{2}=0.2, k_{1}=$ $k_{2}=0.5$, and other parameters are kept the same as our proposed RLC method. The tracking errors $e^{(i)}(i=1,2)$ can also be driven to convergence. However, Fig. 5 shows the obtained control torques $\tau^{(i)}(i=1,2)$ at time interval $[64,72]$ (the time interval $[64,72]$ corresponds to a period of the tracking trajectories). Compared with the performance shown in Fig. 3, they exhibit severe chattering with large amplitude. The control chattering is undesired in practical applications. It is worth pointing out that the tracking accuracy will likely be deteriorated when we try to reduce the chattering.

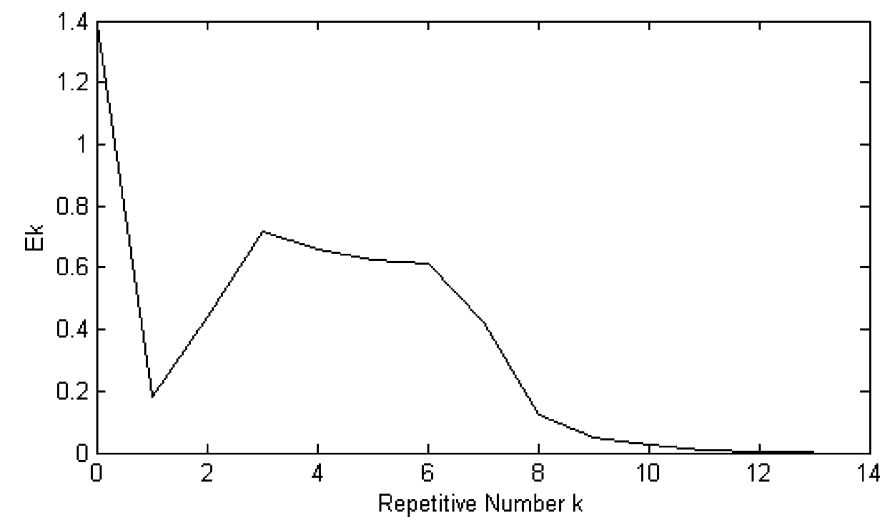

Fig. 4. Norm index $E_{k}$ produced by the proposed RLC method under periodic perturbation.
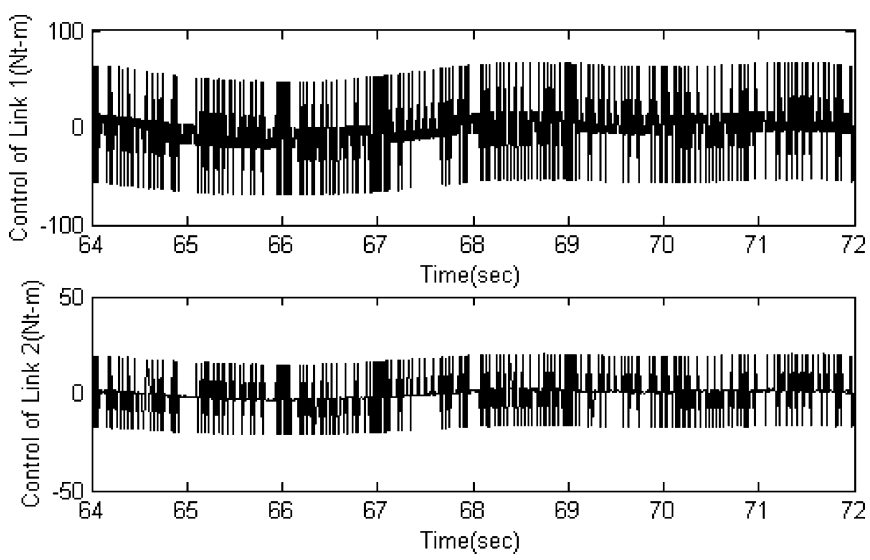

Fig. 5. Control torques at the time interval $[64,72]$ obtained by the VSC technique under periodic perturbation.

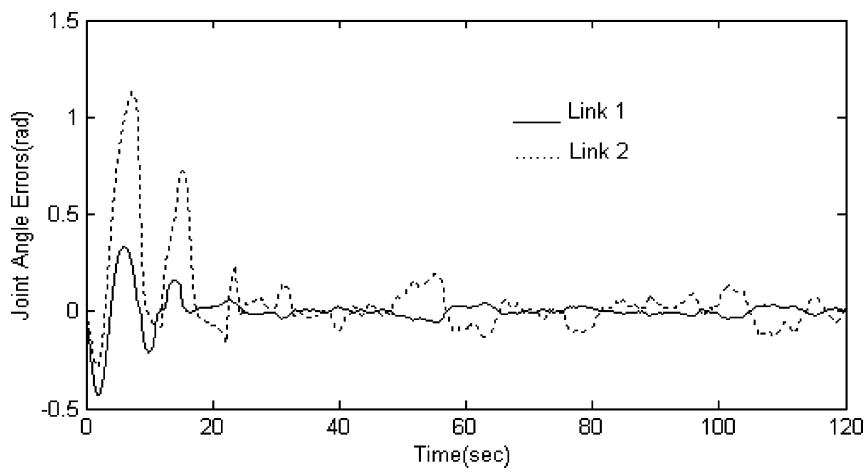

Fig. 6. Joint angle errors obtained by the proposed RLC method under nonperiodic perturbation.

2) The perturbation $w$ is assumed to be nonperiodic given as a serious of random variable with elements uniformly distributed between -1 and 1 . Similar to case 1), the tracking errors $e^{(i)}$ of the joint angles $\theta^{(i)}$ are shown in Fig. 6. It is noticed that the tracking errors are confined to a bounded range after convergence. This demonstrates the robustness of the proposed RLC rule (14).

\section{CONCLUSION}

A new QSM-based RLC approach is designed for MIMO nonlinear uncertain continuous-time systems to address the 
problem of rejecting periodic exogenous disturbances and tracking periodic reference trajectories. The proposed control methodology is useful to the control of industrial robotics, and servo mechanisms, in which periodic signals are commonly experienced. It exhibits an impressive effect of rejecting periodic exogenous disturbances with matching condition when the control systems have tracked periodic reference trajectories. Also, a robust stability of the proposed RLC method against nonperiodic uncertainties and exogenous disturbances is ensured. The RLC rule is derived by applying the modified discrete QSM control in the repetitive direction $k$. In this brief, the proposed RLC method was applied to a robotic arm and compared with the conventional VSC technique. The comparative results demonstrate that the QSM-based RLC approach can avoid the chattering suffered by the conventional VSC technique when tackling the periodic exogenous disturbances.

The proposed RLC approach for nonlinear continuous-time systems is in fact a 2-D nonlinear process. An interesting topic for future study is to investigate the RLC approach for MIMO nonlinear systems using 2-D nonlinear system theory.

\section{ACKNOWLEDGMENT}

The authors would like to thank Dr. W. Guo for his useful discussion. They would also like to thank the Associate Editor and the anonymous referees for their very professional and constructive comments.

\section{REFERENCES}

[1] S. Hara, Y. Yamamoto, T. Omata, and M. Nakano, "Repetitive control system: A new type servo system for periodical exogenous signals," IEEE Trans. Autom. Contr., vol. 33, no. 7, pp. 659-668, Jul. 1988.

[2] M. Sun, Y. Wang, and D. Wang, "Variable-structure repetitive control: A discrete-time strategy," IEEE Trans. Ind. Electron., vol. 52, no. 2, pp. 610-616, Apr. 2005.

[3] J. Ghosh and B. Panden, "Nonlinear repetitive control," IEEE Trans. Automat. Contr., vol. 45, no. 5, pp. 949-954, May 2000.
[4] A. Alleyne and M. Pomykalski, "Control of a class of nonlinear systems subject to periodic exogenous signals," IEEE Trans. Contr. Syst. Technol., vol. 8, no. 2, pp. 279-287, Mar. 2000.

[5] W. Messner, R. Horowitz, W.-W. Kao, and M. Boals, "A new adaptive learning rule," IEEE Trans. Automat. Contr., vol. 36, no. 2, pp. 188-197, Feb. 1991.

[6] B. T. Costic, M. S. de Queiroz, and D. M. Dawson, "A new learning control approach to the active magnetic bearing benchmark system," in Proc. Amer. Contr. Conf., 2000, pp. 2639-2643.

[7] K. Mainali, S. K. Panda, J. X. Xu, and T. Senjyu, "Position tracking performance enhancement of linear ultrasonic motor using iterative learning control," in Proc. 35th Annu. IEEE Power Electron. Specialists Conf., 2004, pp. 4844-4849.

[8] W.-J. Cao and X.-K. Gao, "Repetitive variable structure control of micro-actuators with periodic disturbance and parametric uncertainties," IEEE Trans. Magn., vol. 37, no. 4, pp. 1902-1905, Apr. 2001.

[9] Z. Cao and G. F. Ledwich, "Adaptive repetitive control to track variable periodic signals with fixed sampling rate," IEEE/ASME Trans. Mechatronics, vol. 7, no. 3, pp. 378-384, Sep. 2002.

[10] H. K. Khalil, "Robust servomechanism output feedback controllers for feedback linearizable systems," Automatica, vol. 30, no. 10, pp. 1587-1599, 1994.

[11] R. Horowitz, W. Messner, and J. B. Moore, "Exponential convergence of a learning controller for robot manipulators," IEEE Trans. Autom. Contr., vol. 36, no. 7, pp. 890-894, Jul. 1991.

[12] G. Weiss and M. Hafele, "Repetitive control of MIMO systems using $H^{\infty}$ design," Automatica, vol. 35, no. 7, pp. 1185-1199, 1999.

[13] B. A. Francis and W. M. Wonham, "The internal model principle of control theory," Automatica, vol. 12, no. 5, pp. 457-465, 1976.

[14] W. Gao, Y. Wang, and A. Homaifa, "Discrete-time variable structure control systems," IEEE Trans. Ind. Electron., vol. 42, no. 2, pp. 117-122, Apr. 1995.

[15] C. Milosavljevic, "General conditions for the existence of a quasi sliding mode on the switching hyperplane in discrete variable structure systems," Autom. Remote Contr., vol. 46, no. 3, pp. 307-314, 1985.

[16] A. Bartoszewicz, "Discrete-time quasi-sliding-mode control strategies," IEEE Trans. Ind. Electron., vol. 45, no. 4, pp. 633-637, Aug. 1998.

[17] E. Rogers, K. Galkowski, A. Gramacki, J. Gramacki, and D. H. Owens, "Stability and controllability of a class of linear systems with dynamic boundary conditions," IEEE Trans. Circuits Syst. I, Reg. Papers, vol. 49, no. 2, pp. 181-195, Feb. 2002.

[18] H. Asada and J. J. E. Slotine, Robot Analysis and Control. New York: Wiley, 1986.

[19] W. Gao and J. C. Huang, "Variable structure control of nonlinear systems: A new approach," IEEE Trans. Ind. Electron., vol. 40, no. 1, pp. 45-55, Feb. 1993. 